\title{
Effect of Different Obturation Techniques on Dentinal Tubule Penetrations of MTA Fillapex and AH Plus
}

\author{
Fatma Kermeoğlu' (D), Meltem Küçük' (D), Umut Aksoy' (D), Kaan Orhan² (D) \\ 'Department of Endodontics, Near East University, Faculty of Dentistry, Nicosia, Cyprus \\ ${ }^{2}$ Department of Dentomaxillofacial, Ankara University, Faculty of Dentistry, Ankara, Turkey
}

ORCID iDs of the authors: F.K. 0000-000I-9238-53I2; M.K. 0000-000I-5073-4289; U.A. 0000-000I-728I-508X; K.0. 0000-000I-6768-0I76.

Cite this article as: Kermeoğlu F, Küçük M, Aksoy U, Orhan K. Effect of Different Obturation Techniques on Dentinal Tubule Penetrations of MTA Fillapex and AH Plus. Cyprus J Med Sci. 202I; 6(4): 33I-336.

\section{BACKGROUND/AIMS}

The aim of this in vitro study was to evaluate three different obturation techniques with two different root canal sealers for penetration into dentinal tubules using confocal laser scanning microscopy (CLSM).

\section{MATERIAL and METHODS}

Sixty root canals were prepared and divided into six groups $(n=10)$ : AH Plus + single-cone technique, AH Plus + lateral compaction, AH Plus + continuous-wave technique, MTA Fillapex + single-cone technique, MTA Fillapex + lateral compaction, and MTA Fillapex + continuous wave technique. Following the obturation of the root canals, the specimens were horizontally sectioned, and the sealer penetration percentage, depth, and area were measured at the apical and middle root areas using CLSM analysis.

\section{RESULTS}

The single-cone obturation technique exhibited lower penetration values than the continuous wave and lateral compaction techniques in the AH Plus groups $(P<.05)$. No significant differences were observed between different obturation techniques in MTA Fillapex groups $(P>$.05). MTA Fillapex exhibited significantly higher penetration values than AH Plus in both the apical and the middle third area in terms of depth, area, and percentage $(P<.05)$.

\section{CONCLUSION}

Within the limitations of this in vitro study, MTA Fillapex penetration into the dentinal tubules was not affected by the obturation technique. Using the lateral compaction and continuous wave techniques provided enhanced AH Plus penetration compared to the singlecone technique.

Keywords: Root canal obturation, AH plus, MTA fillapex, obturation techniques, sealer penetration

\section{INTRODUCTION}

The removal of microorganisms and microbial by-products from the root canal system and the prevention of reinfection is the primary objectives of endodontic therapy. The root canal filling is the final step in the classic triad of endodontic therapy: cleaning, shaping, and obturation. The degree of disinfection and integrity of the root canal obturation greatly depend on the cleaning and shaping processes. However, complete eradication of all microorganisms in the radicular space is infeasible or practically almost impossible due to the intricate anatomy of the root canal system and components such as the lateral canals, deltas, isthmuses, and dentinal tubules. ${ }^{2}$ It is well established that many species seen in endodontic infections, such as anaerobic/facultative bacteria and fungi, can easily infiltrate the dentinal tubules. ${ }^{3,4}$ Thus, a hermetic root canal filling has a significant positive effect on clinical outcomes, as it entombs and restricts the surviving microorganisms and microbial by-products within the root canal space. ${ }^{5}$

The most frequently used obturation core material inside the root canal is gutta-percha. Its downside is that it has no adhesive penetration to root canal dentin regardless of the obturation technique used. 6 Sealers plug the gaps between the dentin wall and the core material. They also function as lubricants, thus facilitating the filling of the root canal. The sealers may spread into anatomical irregularities, small and inaccessible areas of the root canal system, and even tubules in the dentin. ${ }^{7}$ 
In endodontic treatment, spreading sealers inside the dentinal tubules is potentially beneficial and, thus, desirable. It provides a reduced interface between the dentin wall and guttapercha, which can enhance the sealing capacity. The obturation material's retention may also be improved as a result of the mechanical joining. Sealers inside the dentinal tubules can also entomb any remaining viable microorganisms, and the chemical constituents of many sealers have antimicrobial properties. ${ }^{8-10}$

AH Plus (Dentsply DeTrey GmbH, Konstanz, Germany) is a paste-paste type material based on epoxy resin. It has been extensively used in endodontic practice. AH Plus has good manipulation characteristics and favorable physical properties, including adhesion to dentin and adequate sealing capability. 11,12 It has been used as a control material in most research on sealers because it has been tested in numerous studies and is considered the gold standard. ${ }^{13,14}$

MTA Fillapex (Angelus Dental Solutions, Londrina, PR, Brazil) root canal sealer is a calcium silicate-based obturation material. It was developed to take advantage of the excellent properties of mineral trioxide aggregate, such as superior biocompatibility, antimicrobial action, and sealing capability. MTA Fillapex consists of mineral trioxide aggregate, salicylate, diluted and natural resins, nanoparticulated silica, and bismuth oxide. ${ }^{15}$

Several techniques have been proposed to achieve hermetic three-dimensional obturation, including lateral compaction (warm or cold), warm vertical compaction (continuous wave), and single-cone obturation. $7,16,17$ The choice of the obturation technique plays an essential role in enhancing the seal, preventing microleakage and filling irregularities within the root canal system. It has been observed that the obturation technique affects the rate of sealer penetration into dentinal tubules. ${ }^{76}$ However, few studies have examined the tubule penetration properties of MTA Fillapex, and, to the best of our knowledge, none of them have evaluated its use with different obturation methods in comparison to AH Plus. Thus, this study aimed to investigate the dentinal tubule penetration of a calcium silicate-based MTA Fillapex sealer compared with $\mathrm{AH}$ Plus using three different obturation methods. The null hypothesis was that the obturation method would not affect the dentinal tubule penetration properties of MTA Fillapex or AH Plus.

\section{Main Points}

- AH Plus sealer penetration into the dentinal tubules was significantly affected by the root canal obturation techniques $(P<.05)$, whereas MTA Fillapex sealer penetration rates were not significantly affected by obturation techniques.

- Regardless of the sealer used, the middle thirds had greater tubule penetration values compared to the apical thirds, in terms of all evaluated parameters.

- The greatest sealer penetration values were found in MTA Fillapex + Continuous Wave group in the middle third area, and the lowest sealer penetration values were obtained in $\mathrm{AH}$ Plus + Single Cone group in apical third area.

\section{MATERIAL and METHODS}

Approval for the study protocol was obtained from the Animal Ethics Committee of the Near East University (2019/66/755).

\section{Preparation of the Teeth}

Sixty extracted mandibular premolar teeth with closed apices were used in this study. Periapical radiographs were obtained from two different aspects (mesiodistal and buccolingual) to verify the presence of a single canal. A dental operating microscope was used to examine the teeth. Teeth with cracks, root fractures, or caries were eliminated. The selected teeth were stored in vials containing thymol solution (0.1\%) until the experimental procedure was initiated. The vertical size of the teeth from the apex was standardized to $14 \mathrm{~mm}$ using a cylindrical diamond bur under water cooling. The working length was determined using a \#10 K file (VDW, Munich, Germany) inserted into the root canal until its tip was extruded from the apical foramen and then positioned I mm short. The root canal of each specimen was instrumented using ProTaper Universal rotary files (Dentsply Maillefer, Ballaigues, Switzerland) to a size of F4 (40/06). A $2 \mathrm{~mL} \mathrm{NaOCl}$ (5.25\%) solution was used to irrigate the root canals after each instrumentation. After instrumentation, all root canals were rinsed with $5 \mathrm{~mL}$ ethylenediaminetetraacetic acid (EDTA; 17\%) to eliminate the residual smear layer. Saline $(5 \mathrm{~mL})$ was used as a final irrigation solution, and sterile F4-sized paper points were used in all root canals. Two layers of nail polish were applied on the outer surface of the specimens, and their apices were sealed with wax.

\section{Experimental Design}

The instrumented root specimens were then randomly divided into six main groups of 10 samples each with the following combinations of obturation materials and techniques:

Group I: AH Plus + single-cone technique

Group 2: AH Plus + lateral compaction

Group 3: AH Plus + continuous-wave technique

Group 4: MTA Fillapex + single-cone technique

Group 5: MTA Fillapex + lateral compaction

Group 6: MTA Fillapex + continuous-wave technique

The AH Plus and MTA Fillapex sealers were mixed labeled with fluorescent rhodamine B isothiocyanate (0.01\%; Merck, Darmstadt, Germany) to allow confocal laser scanning microscopy (CLSM) evaluation. The labeled sealer materials were then inserted into the root canals with a paste carrier (Lentulo Spiral Filler \#35, Malleifer, Baillagues, Switzerland) operating at 300 rpm for 5 seconds.

In the single-cone technique, F4-sized gutta-percha points were coated with AH Plus or MTA Fillapex and placed in each root canal.

In the lateral compaction technique, size 40 master guttapercha points were coated with AH Plus or MTA Fillapex and placed in each root canal. Then, cold lateral compaction was performed using size 20 accessory gutta-percha points and finger spreaders (Thomas, Bourges, France). The procedure was continued until the instrument could not be positioned more than $2 \mathrm{~mm}$ from the coronal orifice. 

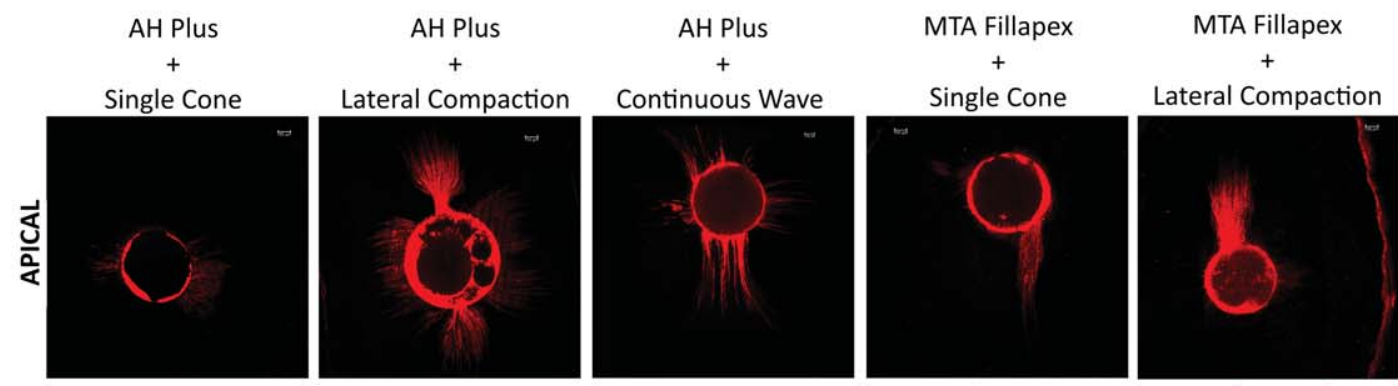

MTA Fillapex
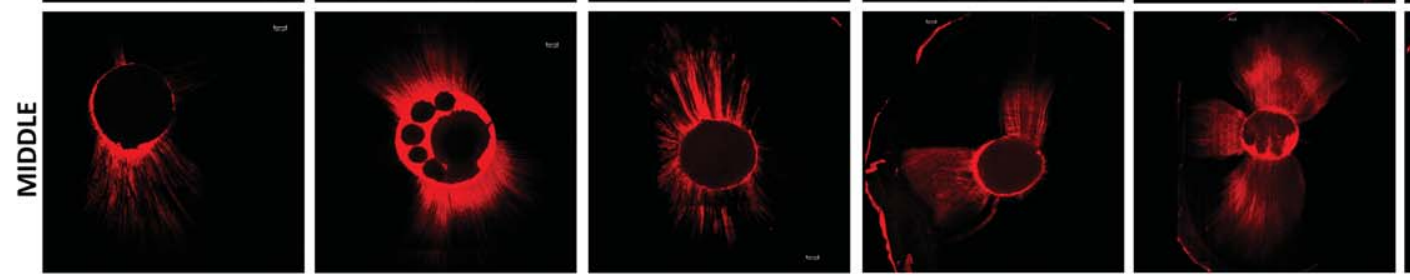

Continuous Wave

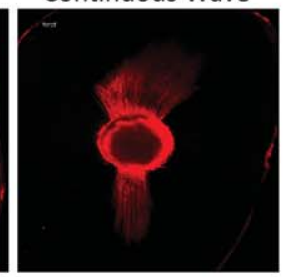

Figure I. Representative CLSM images from each experimental group at the apical third and middle third of the root canal.

In the continuous wave technique, root canal obturations were performed with labeled AH Plus or MTA Fillapex and guttapercha using a Calamus Dual 3D Obturation Device (Dentsply Tulsa Dental, Tulsa, OK, USA) at a temperature of $160^{\circ} \mathrm{C}$ and $60 \%$ flow rate, according to the manufacturer's operation specifications. The master gutta-percha point with the sealer was adapted as in the single-cone technique and removed at the level of the orifice. Then, appropriate sizes of Calamus Electrical Heat Pluggers were used in one continuous motion to remove the gutta-percha $4 \mathrm{~mm}$ short of the working length. The remaining gutta-percha in the root canal was condensed using manual pluggers to obtain a uniformly dense mass at the apical third. A Calamus Flow Obturation Delivery System was used to perform obturation by backfilling the rest of the canal, followed by compaction with a manual plugger. A temporary filling material (Cavit-G, 3M ESPE, Neuss, Germany) was used to seal the specimens' coronal openings. The success of the root canal obturation procedures was confirmed with periapical radiographs. Specimens were then incubated for 2 weeks in $100 \%$ humidity at $37^{\circ} \mathrm{C}$ for a complete setting.

\section{Sectioning and Confocal Laser Scanning Microscopic Analysis of the Roots}

After 2 weeks, the root specimens were vertically mounted on acrylic blocks. Each specimen was transversally cleaved at the middle and apical thirds (5 and $3 \mathrm{~mm}$ from the apex, respectively), and a section approximately $\mathrm{I} \pm 0.1 \mathrm{~mm}$ thick was collected with a low-speed IsoMet saw $(0.3 \mathrm{~mm}$ blade; Buehler, Lake Bluff, IL, USA) under water cooling. Silicon carbide abrasive papers were used to polish the coronal surfaces of the sample slices to remove debris formed during sectioning. The apical surface of each sample slice was mounted onto a sliding glass. The slides were examined using CLSM (Leica TCS SP2, Leica Microsystems, L'Hospitalet de Llobregat, Spain) at $5 \times$ magnification under $\mathrm{Ar} / \mathrm{HeNe}$ laser excitation with a wavelength of $543 \mathrm{~nm}$.

The ImageJ software (National Institutes of Health) was used to perform sealer penetration area, percentage, and depth measurements in digital CLSM images by two blinded operators. The percentage of sealer penetration was calculated by outlining and measuring the parts of the canal circumference, in which sealer penetration was seen and dividing it by the total circumference of the canal wall. The total dentinal penetration area was calculated by measuring the entire root canal and sealer-penetrated areas and subtracting the canal area from the value. The results were recorded in square millimeters $\left(\mathrm{mm}^{2}\right)$. The penetration depth was calculated by measuring the point of maximum penetration from the canal wall and recorded in micrometers $(\mu \mathrm{m})$.

\section{Statistical Analysis}

The data were analyzed using Statistical Package for the Social Sciences (SPSS) version 20.0 (IBM SPSS Corp.; Armonk, NY, USA). The Kruskal-Wallis $\mathrm{H}$ test and T-test were used for overall group comparison at each level. A value of $P<.05$ was considered statistically significant.

\section{RESULTS}

The results are illustrated in Figure I. Representative CLSM images from each experimental group are shown in Figure 2. In the $\mathrm{AH}$ Plus groups, the dentinal tubule penetration depth, area, and percentage were significantly affected by the root canal obturation techniques $(P<.05)$. The single-cone obturation technique exhibited significantly lower penetration values than the continuous wave and lateral compaction techniques $(P<$.05). In the MTA Fillapex groups, the continuous wave technique had the highest penetration depth, area, and percentage values both in the apical and in the middle third area, although the difference was not statistically significant $(P>$ .05). Regardless of the obturation technique used, MTA Fillapex exhibited significantly greater penetration values than $\mathrm{AH}$ Plus in both the apical and the middle third area in terms of depth, area, and percentage $(P<.05)$.

A statistically significant association was found between the different root thirds and the dentinal tubule sealer penetrations $(P<.05)$. The middle third had greater sealer penetration values than the apical third in all evaluated parameters $(P<$ .05).

\section{DISCUSSION}

Several techniques, such as light microscopy, ${ }^{16,17}$ scanning electron microscopy (SEM), $9,18,19 \mathrm{CLSM}^{20-25}$ and microcomputed 


\section{Sealer Penetration Depth $(\mu \mathrm{m})$}

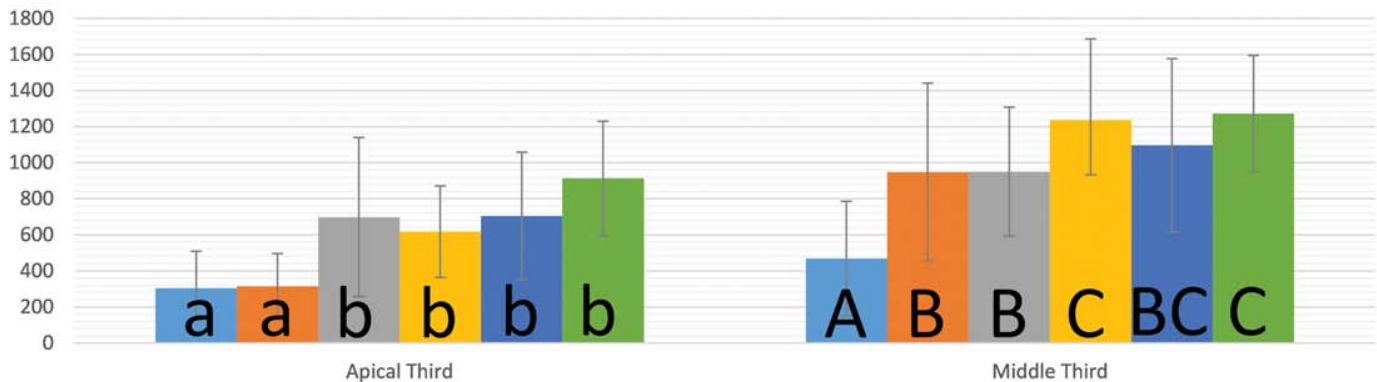

Sealer Penetration Area $(\mathrm{mm} 2)$

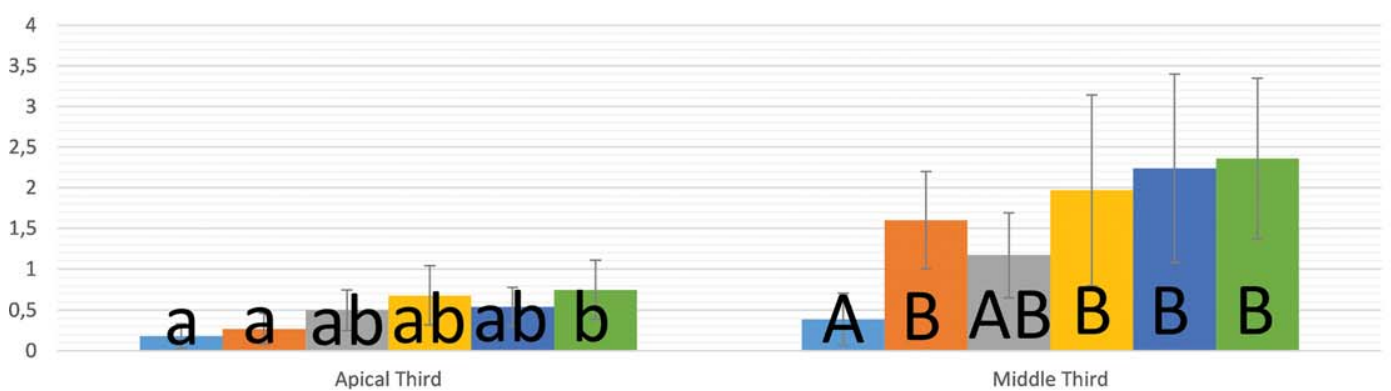

Sealer Penetration Percentage (\%)

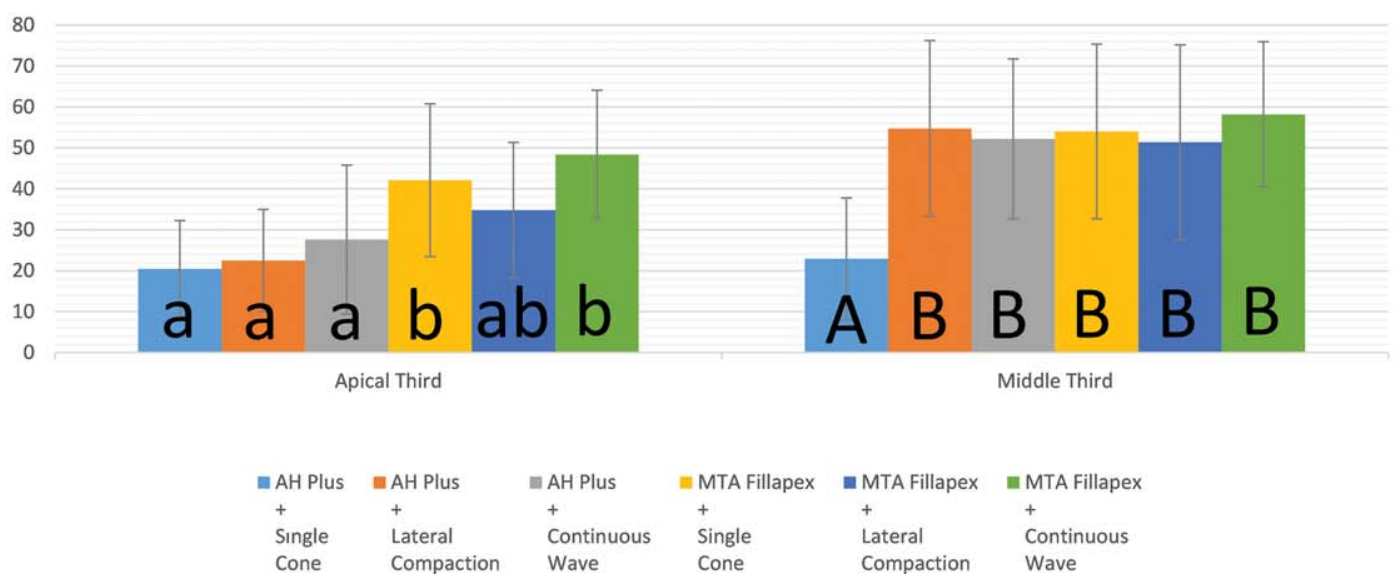

Figure 2. The penetration depth $(\mu \mathrm{m})$, penetration area $\left(\mathrm{mm}^{2}\right)$, and penetration percentage $(\%)$, of AH Plus and MTA Fillapex in different groups at apical third and middle third regions. Different uppercase and lowercase letters in each column indicate statistically significant differences at apical third and middle third $(P<.05)$.

tomography (micro-CT), ${ }^{26}$ have been employed to study sealer penetration in dentinal tubules. SEM imaging has several advantages in exploring the adaptation of sealer materials. It can effectively visualize the dentinal tubules and their contents as well as the dentin-sealer interface, which cannot be achieved with any other method. ${ }^{27}$ On the other hand, CSLM offers several advantages over other conventional methods (SEM and light microscopy) for the study of dental materials. ${ }^{28}$ It allows effective monitoring and analysis of the inward layers under the smear formation without requiring destructive sample processing, thus eliminating the need for technical artifacts due to the loss of tooth structure and sealer material. ${ }^{7,16}$ For this reason, CLSM was selected for this study.
The sealer's penetration capability depends on various factors, including in vivo conditions (smear layer, dentinal tubule density, and diameter, root canal dimensions), as well as such physicochemical properties, such as surface tension, viscosity, solubility, and particle size. 8,9 The smear layer is a critical factor for penetration capability. Smear formation obstructs the penetration of sealers, irrigation solutions, and intracanal medication agents into dentinal tubules and should, therefore, be eliminated with chelating agents. ${ }^{8}$ Previous research has particularly focused on the effect of various irrigation materials and protocols on the penetration ability of sealers. ${ }^{|3,29-3|}$ Since the effect of smear formation was not the main focus of our study, smear layers were removed from all specimens using the same 
$\mathrm{NaOCl}$ and EDTA protocols to increase dentinal permeability. As a result, high penetration values were observed in all groups.

The impact of the various obturation techniques on sealer penetration has received relatively little attention in the literature. Hence, this study aimed to determine the effect of obturation techniques on the penetration capability of two different sealers. The results indicate significant differences in maximum sealer penetration percentage, depth, and area between $\mathrm{AH}$ Plus groups, and consequently, the null hypothesis tested is partially rejected. These findings seem to be consistent with some earlier studies, which found that penetration was affected by both the obturation technique and sealer material. ${ }^{10,21}$ However, contrary results have also been reported. Weis et al. ${ }^{17}$ found that tubule penetration was not affected by different obturation techniques, including continuous wave, cold lateral compaction, and two different carrier-based techniques. Kuçi et al. ${ }^{21}$ observed higher tubule penetration when using MTA Fillapex with the cold lateral compaction technique and $\mathrm{AH} 26$, the predecessor of $\mathrm{AH}$ Plus, with the warm vertical compaction technique. These findings suggest that the effect of the obturation technique on penetration is related to the sealer type. Our results regarding AH Plus reveal that the continuous wave condensation technique exhibits significantly greater values of maximum penetration depth, area, and percentage than the lateral compaction and single-cone techniques, which provides further support to the hypothesis that the effect of the obturation technique on penetration depends on the type of sealer. In contrast to AH Plus, MTA Fillapex groups exhibit similar sealer penetration values when obturated with different techniques. It can be assumed that this difference is due to the behavior of the sealer materials under different physical conditions such as pressure and temperature. Greater $\mathrm{AH}$ Plus tubule penetration is possibly related to a higher flow of the material under pressure and/or its transformation from a paste to a fluid consistency upon warm application during the obturation process, which enables it to penetrate deeper into the dentin tissue.

Regarding the sealer penetration assessment with CLSM, three parameters have been measured in the literature: the percentage of sealer penetration, the maximum and mean penetration depth, and the dentinal tubule penetration area. Most studies have explored the first two parameters based on the method described by Gharib et al. ${ }^{20}$ However, these methods have some limitations. Multiple or single measurements have been performed for the calculation of the deepest sealer penetration. A single or a few measurements for depth may not accurately reflect the actual penetration ability of a sealer. Similarly, assessing the penetration percentage may be insufficient, as it ignores the penetration depth and thickness. To overcome these limitations, recent studies have measured the total dentinal tubule penetration area. $13,32,33$ In this study, the depth and percentage measurements performed in addition to area measurements were aimed at comparing the compatibility between the parameters. The findings suggest that depth, percentage, and area values are largely consistent with each other. For instance, MTA Fillapex exhibits significantly higher penetration values than AH Plus in all three measurements.

The results of this study show that the sealers' dentinal tubule penetration is greater in the middle third section compared with the apical section, regardless of the sealer and technique used. This is in line with previous studies that investigated various sealers and obturation techniques. ${ }^{20,21}$ A few explanations can be proposed for this observation. First, it is known that dentinal tubule diameters are smaller in the apical area and that the tubule density also decreases toward the apex. ${ }^{34}$ Second, more sclerotic dentin is more present at the apical level of the root. ${ }^{35} \mathrm{~A}$ third factor is a difficulty eliminating the smear layer in the apical area. ${ }^{36}$ These factors affect the dentin's permeability and, consequently, the sealer's penetration rate. The interesting difference observed in the penetration ratios between buccolingual direction and mesiodistal direction may be explained by the effect of increased dentinal sclerosis on the lateral sides (distal and mesial) of the root dentin. This has been termed the "butterfly effect" because its shape resembles a butterfly, as seen in crosssection images of root dentin. ${ }^{21,27}$ The findings of the present study are clinically significant owing to the fact that all evaluated parameters that are the tubule penetration depth, percentage, and area by sealers may affect filling quality and impermeability of the root canal system and prevent reinfection., 8,22 However, the sealer penetration into dentinal tubules was reported not to be directly related to the apical seal in a recent study by DeDeus et al. ${ }^{37}$ A limitation of the current study was the impossibility of standardization of the amount and distribution of sclerotic dentin despite the careful sample selection. ${ }^{38}$ Irregular secondary dentin may influence sealer penetration.

Clinicians' understanding of the features of the endodontic sealer materials improves the treatment outcome. The continuous wave and lateral compaction techniques are more effective than the single-cone technique with $\mathrm{AH}$ Plus. The tubular penetration of MTA Fillapex is not affected by different obturation techniques. Regardless of the technique used, MTA Fillapex penetrates the dentinal tubules significantly better than AH Plus, suggesting that, in terms of filling durability, it might be beneficial to obturate the root canal with MTA Fillapex.

Ethics Committee Approval: Ethical committee approval was reccieved from the Animal Ethics Committee of the Near East University (2019/ $66 / 755)$.

\section{Informed Consent: N/A}

Peer-review: Externally peer-reviewed.

Author Contributions: Concept - F.K., M.K., U.A., K.O.; Design - F.K., M.K., U.A., K.O.; Supervision - F.K., U.A., K.O.; Resources - F.K., M.K.; Materials F.K., M.K.; Data Collection and/or Processing - M.K., X.X.; Analysis and/ or Interpretation - M.K., U.A.; Literature Search - U.A., K.O.; Writing Manuscript - U.A., K.O.; Critical Review - U.A., K.O.

Acknowledgments: The authors would like to thank Ms. Gulsen Ozduran for assisting with the statistical analysis. This study was not supported by fundings.

Conflict of Interest: The authors have no conflicts of interest to declare.

Financial Disclosure: The authors declared that this study has received no financial support.

\section{REFERENCES}

I. Zehnder M. Root canal irrigants. J Endod. 2006;32(5):389-398. [CrossRef]

2. Peters LB, Van Winkelhoff AJ, Buijs JF, Wesselink PR. Effects of instrumentation, irrigation and dressing with calcium hydroxide on 
infection in pulpless teeth with periapical bone lesions. Int Endod J. 2002;35(I):I3-2I. [CrossRef]

3. Love RM. Enterococcus faecalis-A mechanism for its role in endodontic failure. Int Endod J. 2001;34(5):399-405. [CrossRef]

4. Love RM, Jenkinson HF. Invasion of dentinal tubules by oral bacteria. Crit Rev Oral Biol Med. 2002;13(2):17|-183. [CrossRef]

5. Whitworth J. Methods of filling root canals: Principles and practices. Endod Top. 2005;12(I):2-24. [CrossRef]

6. Evans JT, Simon JH. Evaluation of the apical seal produced by injected thermoplasticized gutta-percha in the absence of smear layer and root canal sealer. J Endod. 1986;12(3):100-107.

7. Jeong JW, DeGraft-Johnson A, Dorn SO, Di Fiore PM. Dentinal tubule penetration of a calcium silicate-based root canal sealer with different obturation methods. J Endod. 2017;43(4):633-637. [CrossRef]

8. Kokkas AB, Boutsioukis AC, Vassiliadis LP, Stavriaos CK. The influence of the smear layer on dentinal tubule penetration depth by three different root canal sealers: An in vitro study. J Endod. 2004;30(2):I00-102. [CrossRef]

9. Mamootil K, Messer HH. Penetration of dentinal tubules by endodontic sealer cements in extracted teeth and in vivo. Int Endod J. 2007;40(I):873-88I. [CrossRef]

10. Chandra SS, Shankar P, Indira R. Depth of penetration of four resin sealers into radicular dentinal tubules: A confocal microscopic study. J Endod. 2012;38(I0):1412-1416. [CrossRef]

II. Marin-Bauza GA, Rached-Junior FJA, Sousa-Gabriel AE, et al. Physicochemical properties of methacrylate resin-based root canal sealers. J Endod. 2010;36(9):1531-1536. [CrossRef]

12. Marciano MA, Guimarães BM, Ordinola-Zapata R, et al. Physical properties and interfacial adaptation of three epoxy resin-based sealers. J Endod. 2011;37(I0):1417-142I. [CrossRef]

13. Akcay M, Arslan H, Durmus N, Mese M, Capar ID. Dentinal tubule penetration of AH Plus, iRoot SP, MTA fillapex, and guttaflow bioseal root canal sealers after different final irrigation procedures: $A$ confocal microscopic study. Lasers Surg Med. 2016;48(I):70-76. [CrossRef]

14. El Hachem R, Khalil I, Le Brun G, et al. Dentinal tubule penetration of AH Plus, BC Sealer and a novel tricalcium silicate sealer: A confocal laser scanning microscopy study. Clin Oral Invest. 2019;23(4):187|-1876. [CrossRef]

15. Torabinejad M, Parirokh M. Mineral trioxide aggregate: A comprehensive literature review-Part II: Leakage and biocompatibility investigations. J Endod. 2010;36(2):190-202. [CrossRef]

16. De Deus GA, Gurgel-Filho ED, Maniglia-Ferreira C, Coulinho-Filho T. The influence of filling technique on depth of tubule penetration by root canal sealer: A study using light microscopy and digital image processing. Aust Endod J. 2004;30(I):23-28. [CrossRef]

17. Weis MV, Parashos $\mathrm{P}$, Messer $\mathrm{HH}$. Effect of obturation technique on sealer cement thickness and dentinal tubule penetration. Int Endod J. 2004;37(I0):653-663. [CrossRef]

18. Kouvas V, Liolios E, Vassiliadis I, Parissis-Messimeris S, Boutsioukis A. Influence of smear layer on depth of penetration of three endodontic sealers: An SEM study. Endod Dent Traumatol. 2007;|14(4):19|-195. [CrossRef]

19. Balguerie E, van der Sluis L, Vallaeys K, Gurgel-Georgelin M, Diemer $F$. Sealer penetration and adaptation in the dentinal tubules: A scanning electron microscopic study. J Endod. 20II;37(II):1576-1579. [CrossRef]

20. Gharib SR, Tordik PA, Imamura GM, Baginski TA, Goodell GG. A confocal laser scanning microscope investigation of the epiphany obturation system. J Endod. 2007;33(8):957-961. [CrossRef]

21. Kuçi A, Alaçam T, Yavaş O, Ergul-Ulger Z, Kayaoglu G. Sealer penetration into dentinal tubules in the presence or absence of smear layer: A confocal laser scanning microscopic study. J Endod. 2014;40(I0):1627-1631. [CrossRef]

22. Moon YM, Shon WJ, Baek SH, Bae KS, Kum KY, Lee W. Effect of final irrigation regimen on sealer penetration in curved root canals. J Endod. 2010;36(4):732-736. [CrossRef]

23. Kok D, Da Rosa RA, Barreto MS, et al. Penetrability of $A H$ plus and MTA fillapex after endodontic treatment and retreatment: A confocal laser scanning microscopy study. Microsc Res Tech. 2014;77(6):467-47I. [CrossRef]

24. Amoroso-Silva PA, Guimarães BM, Marciano MA, et al. Microscopic analysis of the quality of obturation and physical properties of MTA Fillapex. Microsc Res Tech. 2014;77(12):1031-1036. [CrossRef]

25. Gunes B, Yeter KY, Terlemez A, Seker B, Altay Y. Dentinal tubule penetration of endodontic sealers after nonthermal plasma treatment: A confocal laser scanning microscopy study. Microsc Res Tech. 2019;82(6):903-908. [CrossRef]

26. Huang $Y$, Orhan K, Celikten B, Orhan Al, Tufenkci P, Sevimay S. Evaluation of the sealing ability of different root canal sealers: $A$ combined SEM and micro-CT study. J Appl Oral Sci. 2018;15(26):e20160584.

27. Russell A, Friedlander L, Chandler N. Sealer penetration and adaptation in root canals with the butterfly effect. Aust Endod J. 2018;44(3):225-234. [CrossRef]

28. Moodley D, Grobler SR, Rossouw RJ, Oberholzer TG, Patel N. in vitro evaluation of two adhesive systems used with compomer filling materials. Int Dent J. 2000;50(6):400-406. [CrossRef]

29. Tuncer AK, Tuncer S. Effect of different final irrigation solutions on dentinal tubule penetration depth and percentage of root canal sealer. J Endod. 2012;38(6):860-863. [CrossRef]

30. Aksel H, Küçükkaya Eren S, Puralı N, Serper A, Azim A. Efficacy of different irrigant protocols and application systems on sealer penetration using a stepwise CLSM analysis. Microsc Res Tech. 2017;80(12):I323-I327. [CrossRef]

31. Aksel H, Arslan E, Puralı N, Uyanık O, Nagaş E. Effect of ultrasonic activation on dentinal tubule penetration of calcium silicate-based cements. Microsc Res Tech. 2019;82(5):624-629. [CrossRef]

32. Akcay M, Arslan H, Mese M, Durmus N, Capar ID. Effect of photoninitiated photoacoustic streaming, passive ultrasonic, and sonic irrigation techniques on dentinal tubule penetration of irrigation solution: A confocal microscopic study. Clin Oral Invest. 2017;2I(7):2205-2212. [CrossRef]

33. Aydın ZU, Ozyürek T, Keskin B, Baran T. Effect of chitosan nanoparticle, QMix, and EDTA on TotalFill BC sealers' dentinal tubule penetration: A confocal laser scanning microscopy study. Odontology. 2019;107(I):64-7I. [CrossRef]

34. Carrigan PJ, Morse DR, Furst ML, Sinai IH. A scanning electron microscopic evaluation of human dentinal tubules according to age and location. J Endod. 1984;10(8):359-363. [CrossRef]

35. Ribeiro RG, Marchesan MA, Silva RG, Sousa-Neto MD, Pécora JD. Dentin permeability of the apical third in different groups of teeth. Braz Dent J. 2010;21(3):216-219. [CrossRef]

36. Blank-Gonçalves LM, Nabeshima CK, Martins GH, de Lima Machado ME. Qualitative analysis of the removal of the smear layer in the apical third of curved roots: Conventional irrigation versus activation systems. J Endod. 2011;37(9):I268-1271. [CrossRef]

37. De-Deus $G$, Brandão $M$, Leal F, et al. Lack of correlation between sealer penetration into dentinal tubules and sealability in nonbonded root fillings. Int Endod J. 2012;45:642-65I. [CrossRef]

38. Jardine AP, Da Rosa RA, Santini MF, et al. The effect of final irrigation on the penetrability of an epoxy resin-based sealer into dentinal tubules: A confocal microscopy study. Clin Oral Invest. 2016;20:117-123. [CrossRef] 